\title{
Three-dimensional topology optimized electrically-small conformal antenna
}

\author{
Erentok, Aycan; Sigmund, Ole
}

Published in:

IEEE Antennas and Propagation Society International Symposium, 2008. AP-S 2008.

Link to article, DOI:

10.1109/APS.2008.4619200

Publication date:

2008

Document Version

Publisher's PDF, also known as Version of record

Link back to DTU Orbit

Citation (APA):

Erentok, A., \& Sigmund, O. (2008). Three-dimensional topology optimized electrically-small conformal antenna. In IEEE Antennas and Propagation Society International Symposium, 2008. AP-S 2008. (pp. 1-4). IEEE. https://doi.org/10.1109/APS.2008.4619200

\section{General rights}

Copyright and moral rights for the publications made accessible in the public portal are retained by the authors and/or other copyright owners and it is a condition of accessing publications that users recognise and abide by the legal requirements associated with these rights.

- Users may download and print one copy of any publication from the public portal for the purpose of private study or research.

- You may not further distribute the material or use it for any profit-making activity or commercial gain

- You may freely distribute the URL identifying the publication in the public portal

If you believe that this document breaches copyright please contact us providing details, and we will remove access to the work immediately and investigate your claim 


\title{
Three-dimensional Topology Optimized Electrically-Small Conformal Antenna
}

\author{
Aycan Erentok $^{(1)^{*}}$ and Ole Sigmund ${ }^{(1)}$ \\ (1) Department of Mechanical Engineering, Section for Solid Mechanics, \\ Technical University of Denmark, DK-2800 Lyngby, Denmark \\ E-mail: aer@mek.dtu.dk, sigmund@mek.dtu.dk
}

\section{Introduction}

The design of electrically small antennas has been a research interest to the community since the 1940s due to their potential impact in many communication systems. An optimal electrically small antenna design that can approach the fundamental limits requires clever design configurations to reconcile several contradictory radiation properties of electrically small antennas, e.g., with the reduction of the antenna size, the radiation efficiency and operational bandwidth of the radiator decreases and there is a high impedance mismatch relative to the characteristic impedance of the feeding network. There has been a wide variety of approaches to achieve this goal including optimal packing of resonant antenna elements using natural geometrical configurations [1], nonintuitive structures generated by evolutionary optimization methods [2], and recently proposed theoretical metamaterial-based electrically small antenna systems [3].

The topology optimization method is a well-established gradient-based optimization method that determines its optimization direction using a sensitivity analysis with no restriction on resulting topologies. Evolutionary or other semi-random approaches require a large number of function evaluations even for small number of design elements making these optimization methods difficult to implement in 3D optimization problems. Topology optimization has been successfully implemented in structural designs and to design materials with extreme properties including tailored 'exotic' materials with negative Poisson's ratios and photonic crystal structures [4]. Recently, the topology optimization method has been successfully implemented to design dielectric substrates producing compact antennas with improved antenna performances [5]. In addition, combined conductor shape and material optimization was also reported to achieve further antenna performance enhancements [6].

This paper introduces a three-dimensional (3D) conductor-based, conformal, electrically small antenna (ESA) that is obtained using the topology optimization method. The topology optimization method uses an iterative optimization scheme to distribute a certain amount of conductive material inside a 3D arbitrary shaped design domain such that the material layout defines an electrically small conformal radiator fed by a coaxialcable over a ground plane that maximizes the far-field radiated power. A partially cut hemispherical shell volume is selected as the 3D design domain to model a nonsymmetric and conformal 3D ESA. The preliminary research investigations demonstrate that the conformal ESA design has a radiation efficiency of approximately $80 \%$. The computed reflection coefficient at the operation frequency is $\mathrm{S}_{11}=-24.6 \mathrm{~dB}$. It is important to note that this optimization methodology is cable of optimizing the desired antenna parameters for any shape of the design domain. The optimization capability of this methodology is mainly limited by the resolution of the mesh size. 


\section{Design Methodology}

The proposed conformal electrically small antenna design bases on the COMSOL Multiphysics commercial finite-element software package. The 3D finite-element model is used both for the solution of the 3D Maxwell's problem and for the sensitivity analysis required by the optimization algorithm. The software package also provides the geometry setup of a given problem, mesh generation and post-processing tools that accelerates a given design cycle.

A single design variable $x_{i}$ is assigned to each corresponding finite element and it represents the density of that finite element in the design domain:

$$
x_{i} \in \mathbb{R} \mid 0 \leq x_{i} \leq 1 \text { for } \mathrm{i}=1,2,3, N,
$$

where $N$ is the total number of designs variables determined by the number of tetrahedras that are used to mesh the specified design domain. A suitable function relating the surface conductivity value, $\sigma$, to the design variable is obtained from [5]

$$
\sigma=\sigma_{\min }\left(\sigma_{\max } / \sigma_{\min }\right)^{x_{i}}
$$

where $\sigma_{\min }=10^{-4}$ and $\sigma_{\max }=10^{6}$ corresponding to air and the conductor material, respectively. Numerical investigations have suggested a different set of $\sigma_{\min }$ and $\sigma_{\max }$ values than the ones reported in [5] for this particular design. Preferably, the optimal design should have no finite elements at the intermediate conductivity values as these design variables cannot be easily manufactured. The topology optimization method is applied to the specified 3D conformal, non-symmetric design domain to maximize the far-field radiated power of the designed antenna structure at a single frequency with respect to a given volume constraint. The cost function can thus be formulated as

$$
\begin{gathered}
\max \left(E_{x} \times E_{x}{ }^{*}+E_{y} \times E_{y}{ }^{*}+E_{z} \times E_{z}^{*}\right) /(2 \times \eta) \\
\text { subject to a material volume constraint: } \sum_{i=1}^{N} x_{i} \cdot V_{i} \leq V^{*}
\end{gathered}
$$

where $E_{x}^{*}, \mathrm{~V}_{\mathrm{i}}$ and $\eta$ are the conjugate $\mathrm{x}$-component of the electric-field at the radiation boundary, material volume of the $i^{\text {th }}$ finite element and free-space impedance, respectively. The optimization problem given in (3) is initiated using a solid conductor material, $x_{i}=1$ for all finite elements, with a $60 \%$ volume constraint. The cost function and sensitivities with respect to the design variables are solved using an iterative procedure, where a popular mathematical programming tool method of moving asymptotes (MMA) [7] is used to update the density of each finite element. A filter is also added to ensure mesh-independency [4]. The filter radius is set to the maximum dimension of the finite element in the design domain. The optimization cycle is stopped when design changes between successive iterations goes below a given tolerance. The flow of computations for the optimization cycle is demonstrated in Fig. 1a.

The finite element model of the 3D conformal ESA is constructed using COMSOL tools as shown in Fig. 2a. All the sub-domains are assigned as free-spaces except the partially cut hemispherical shell domain, e.g., Eqn. 2 is assigned to the conductivity value of the 
design domain. The coaxial feed is modelled using PEC boundaries and excited with $1 \mathrm{~W}$ input power, where a coaxial port option is used for the excitation. The radius of the outer conductor $r_{b}$ was set equal to $r_{b}=r_{a} \times 2.301=2.301 \mathrm{~mm}$ to achieve a characteristic impedance of the coax TEM mode of $50 \Omega$. The partially cut hemispherical shell design domain is $2 \mathrm{~mm}$ thick, and the radius of the outer layer is $79 \mathrm{~mm}$. The hemispherical part that is larger than the $\mathrm{x}=9 \mathrm{~mm}$ plane is removed to obtain the desired non-symmetric $3 \mathrm{D}$ geometry. The target frequency of interest here is $f=300 \mathrm{MHz}$ and thus, the maximum dimension of the antenna must be smaller than $a=79.58 \mathrm{~mm}$ to meet the electrically small antenna criterion [1]. Figure 2 demonstrates the optimized 3D conductor-based, conformal, electrically small antenna that is obtained using topology optimization method. Our initial findings demonstrate that the topology optimization method produced a conformal ESA design that has a radiation efficiency of approximately $80 \%$ at $300 \mathrm{MHz}$. The reflection coefficient is $\mathrm{S}_{11}=-24.6 \mathrm{~dB}$. In order to produce a purely conductor-based antenna design, not including any intermediate density values, the optimized design variables that have density values less than $57 \%$ are designated as air, and the rest of the material distribution is defined to be the copper material. We are currently investigating how to accurately compute the BW and Q values of this conformal ESA design. Note that the optimized conductor distribution requires a post-processing step to realize a physical antenna design, and we are also studying different topology optimization parameters to produce smoother/easier-to-manufacture antenna designs.

\section{Conclusions}

A three-dimensional (3D) conductor-based conformal electrically small antenna is obtained using a topology optimization method. The optimization method distributes a certain amount of conductive material to a designated design domain such that the material layout defines an electrically small radiator fed by a coaxial cable over a ground plane. Preliminary investigations show that topology optimization method produced a conformal ESA design that has a radiation efficiency of approximately $80 \%$ at $300 \mathrm{MHz}$.

\section{Acknowledgments}

This work received support from the Danish Agency for Science, Technology and Innovation through the grant "Topology Optimization of Electromagmetic Metamaterials for Miniaturization of Wireless Communication Antennas (TopAnt)" and an Eurohorcs/ESF European Young Investigator Award(EURYI, www.esf.org/euryi). Also, the authors wish to thank professors Olav Breinbjerg, Niels Asger Mortensen, Oleksiy Kim and Samel Arslanagic from the TopAnt group for inspiring discussions on the presented work.

\section{References}

[1] S. R. Best, "The radiation properties of electrically small folded spherical helix antennas," IEEE Trans. Antennas Propag., Vol. 52(4), pp. 953-960, Apr. 2004

[2] E. E. Altshuler, "Electrically small self-resonant wire antennas optimized using a genetic algorithm," IEEE Trans. Antennas Propagat., Vol. AP-50(3), pp. 297-300, Mar. 2002

[3] R. W. Ziolkowski and A. Erentok, "Metamaterial-based efficient electrically small antennas," IEEE Trans. Antennas Propagat., Vol. 54, pp. 2113-2130, July 2006 
[4] Bendsøe M. P. and Sigmund O., Topology Optimization -Theory, Methods and Applications, Springer, Berlin, 2003

[5] G. Kiziltas, "Dielectric material optimization of filters and antennas using SIMP," Ph.D. Thesis, University of Michigan, Ann Arbor, May 2003

[6] G. Kiziltas, "Concurrent design of conductor shape and artificial material composites for RF applications," 2006 IEEE AP-S/URSI/AMEREM Symposium, p. 430, July 914, 2006, Albuquerque, NM, USA

[7] K. Svanberg, "The method of moving asymptotes- A new method for structural optimization,” Int. J. Numer. Methods Eng. Vol. 24, pp. 359-373, 1987

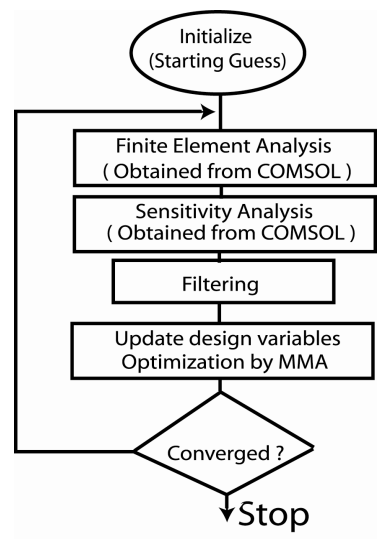

(a)

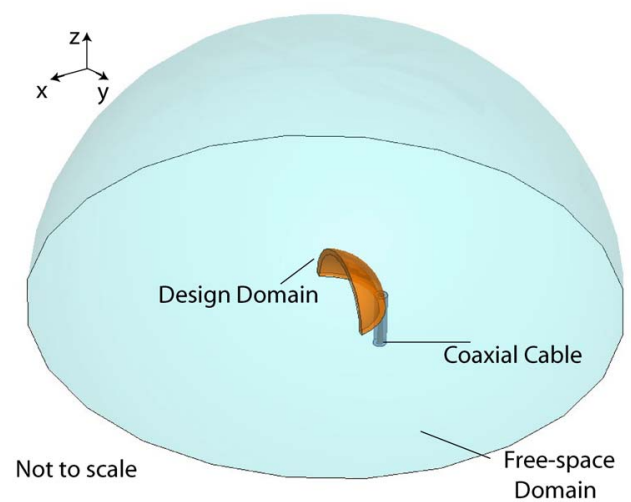

(b)

Fig. 1 (a) The flow of computations for the optimization cycle and (b) COMSOL Multiphysic finite element model of the conformal antenna fed by a coaxial-cable

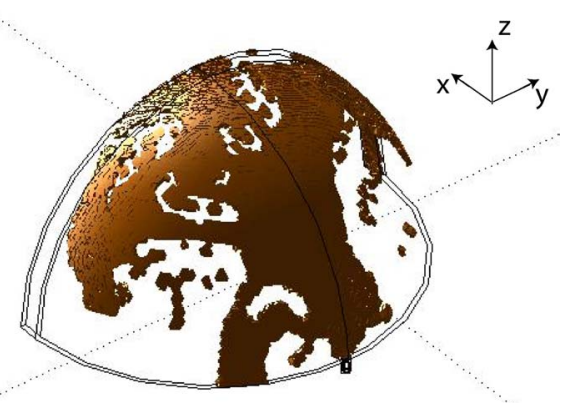

(a)

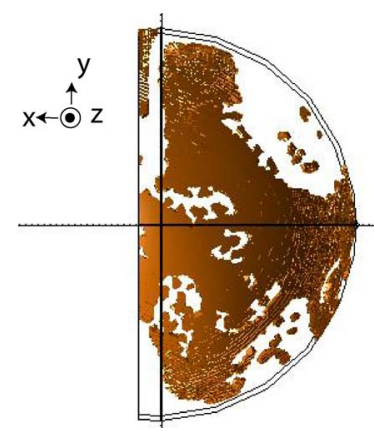

(b)

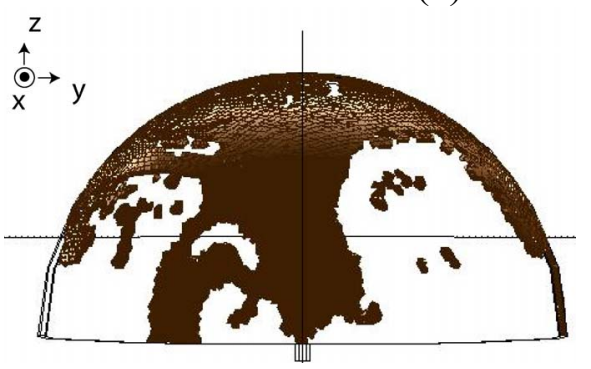

(c)

Fig. 2 The topology optimized 3D conductor-based conformal electrically small antenna (ESA) design at $300 \mathrm{MHz}$ (a) xyz-view, (b) xy-plane view and (c) yz-plane view. The finite elements that have density values smaller than $57 \%$ are designated to be air, and the rest are assigned to be the copper material, e.g., $\sigma=5.998 * 10^{7} \mathrm{~S} / \mathrm{m}$ 